\title{
CARACTÉRISATION DES PANACHES INDUSTRIELS par imagerie hyperspectrale
aéroportée
}

\author{
Xavier BRIOTTET \\ Pierre-Yves FOUCHER \\ Rodolphe MARION \\ Adrien DESCHAMPS \\ ONERA/DOTA, Chemin de la \\ Hunière, 91120 Palaiseau \\ CEA/DAM/DIF, Bruyères-le-Châtel, \\ 91290 Arpajon \\ xavier.briottet@onera.fr
}

La caractérisation des aérosols et des gaz produits par l'homme est un enjeu majeur pour la société car ces composants ont un impact direct sur la santé et le climat. Plusieurs techniques de caractérisation existent mais la télédétection aéroportée est une réponse potentiellement adaptée pour l'étude de ces sources si l'on veut avoir accès à leur expansion spatiale. De plus, l'imagerie hyperspectrale concernant tout le domaine optique, elle permet de couvrir l'ensemble des besoins nécessaires à la détection et la caractérisation des aérosols et des gaz.

global ainsi que sur la formation de l'ozone de pollution troposphérique. L'estimation précise de la quantité de gaz émise par une usine ou de la concentration et des propriétés des aérosols rejetés par une industrie à partir de données satellites ou aériennes permettrait à la fois d'améliorer les méthodes de suivi de pollution et les prévisions de la qualité de l'air, et d'affiner les facteurs d'émissions nécessaires aux modèles climatologiques.

En France, l'INERIS (Institut national de l'environnement industriel et des risques) publie chaque année le Registre français des émissions polluantes recensant les données fournies par les exploitants d'installations industrielles, de stations d'épuration et d'élevages concernant leurs émissions dans l'air, l'eau et le sol. Ces informations importantes restent néanmoins limitées au niveau de la source de pollution et ne donnent pas une vision globale de cette pollution et de son extension. Il apparaît ainsi nécessaire de mettre en place des méthodes pour construire une cartographie de la pollution.

Parmi les différents outils de télédétection, l'imagerie hyperspectrale, ou spectro-imagerie, a un potentiel particulièrement intéressant dans le cadre de la caractérisation d'effluents à une échelle aussi locale qu'un site industriel.

Cette technique (figure 1) permet d'imager le signal incident (luminance spectrale) dans un grand nombre de bandes spectrales étroites et contiguës. La luminance enregistrée est ainsi caractéristique du paysage observé et de l'atmosphère présente (intégrant en particulier la contribution du panache industriel). Les deux principales classes de mesures spectrales reposent sur l'utilisation, soit d'un réseau soit d'un interféromètre.

Ainsi, une telle technique permet, à partir d'une unique image, d'estimer conjointement les cartes de concentration des gaz et des aérosols. De plus, la bonne résolution spatiale des images hyperspectrales (de l'ordre du mètre pour les images aéroportées comme Hymap, à quelques décamètres pour les images satellites comme les futurs systèmes EnMap ou HYPXIM) permet l'étude des effluents au plus proche de leur source d'émission. La couverture spectrale de ces instruments $(0,4$ à $2,5 \mu \mathrm{m})$ permet de caractériser les aérosols et quelques 
gaz $\left(\mathrm{CO}_{2}, \mathrm{CH}_{4}, \mathrm{NO}_{2}\right)$. Afin de caractériser de manière complète les effluents gazeux une couverture spectrale plus large étendue au domaine infrarouge thermique (3-12 $\mu \mathrm{m})$ est requise: dans ce domaine on observe en effet les signatures de la majorité des gaz plus complexes comme le $\mathrm{SO}_{2}$, les $\mathrm{COV}$... S'il existe différents capteurs couvrant partiellement le domaine optique, à ce jour seul l'imageur hyperspectral Sysiphe [2], développé par l'ONERA avec le soutien de la DGA, couvre l'ensemble du domaine spectral 0,4 à $12 \mu \mathrm{m}$ avec la même résolution spatiale.

Ce document va décrire les méthodes de caractérisation des aérosols anthropiques puis des gaz à partir d'imagerie hyperspectrale.

\section{Caractérisation des aérosols}

\section{Caractéristiques des aérosols}

Les aérosols atmosphériques sont des particules fines en suspension dans les basses couches de l'atmosphère. Ils peuvent provenir de sources naturelles (poussières soulevées par le vent, embruns océaniques et particules provenant de feux de biomasse ou d'émissions volcaniques) ou anthropiques (émissions de véhicules, rejets industriels, chauffage au bois).
Ils sont définis par leur composition chimique ou minéralogique (caractérisée par leur indice de réfraction), leur taille (granulométrie), leur forme et leur concentration. C'est ce que l'on appelle les propriétés microphysiques d'une particule.

En télédétection optique, l'impact radiatif des aérosols s'exprime par leurs propriétés optiques. Ces dernières traduisent leur interaction avec le rayonnement et sont donc les propriétés observables sur les images acquises par télédétection. Elles sont au nombre de trois: l'épaisseur optique, l'albédo de diffusion simple et la fonction de phase. L'épaisseur optique quantifie le pouvoir d'extinction qu'a un milieu: elle est donc principalement reliée à la concentration en particules, mais dépend également de leur rayon et de leur pouvoir d'extinction, donc de leur nature. L'albédo de diffusion simple caractérise l'importance de la contribution de la diffusion sur l'extinction et est donc lié à la nature de l'aérosol, mais également à sa granulométrie. Enfin, la fonction de phase caractérise le comportement angulaire de la diffusion et est liée à la forme et à la taille des aérosols.

Pour des particules sphériques, la théorie de Mie permet le passage des propriétés microphysiques aux propriétés optiques.

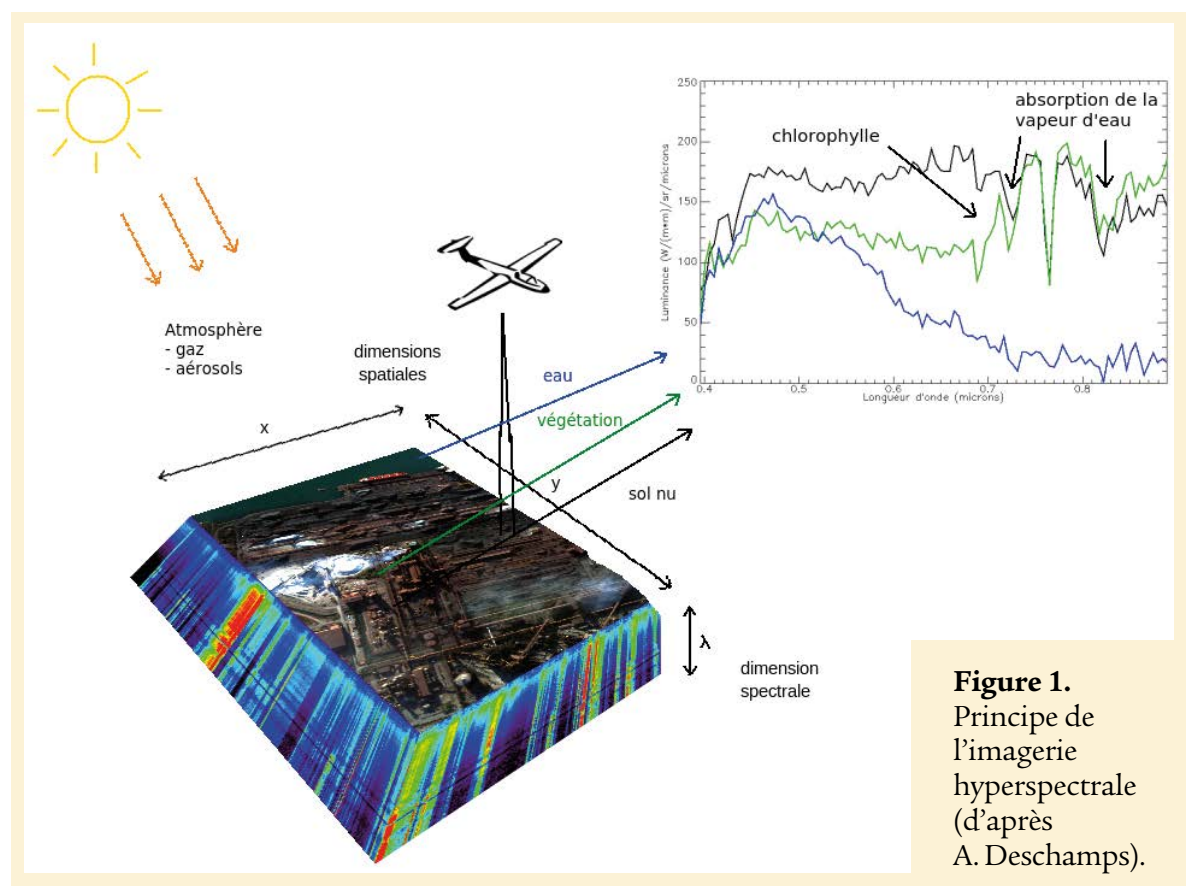

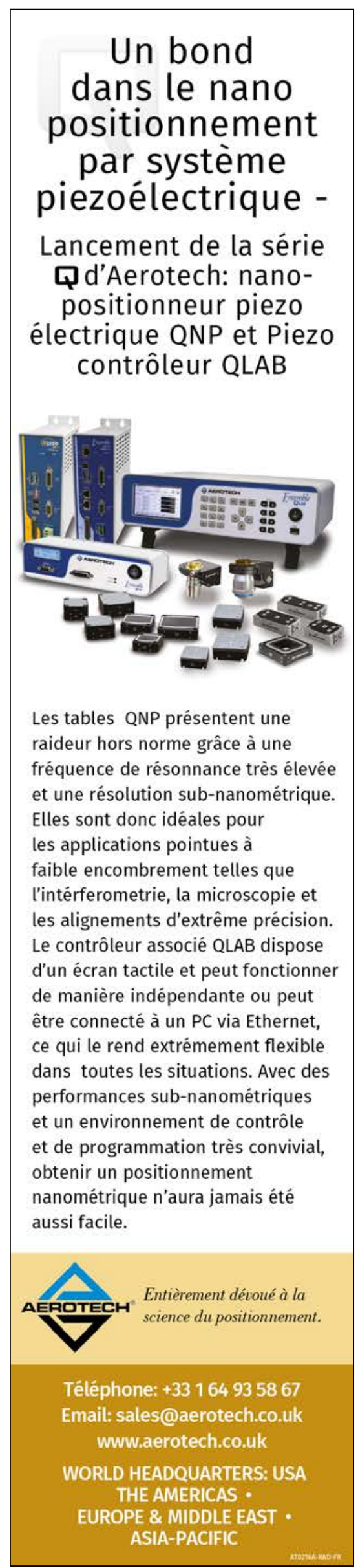



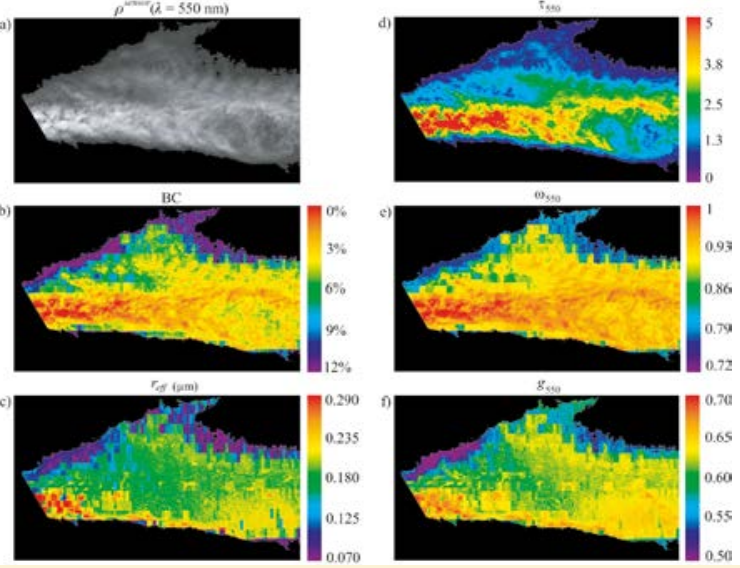

Figure 2. Cartes des propriétés microphysiques et optiques à $550 \mathrm{~nm}$ estimées pour le panache de feux de biomasse du Quinault par la méthode L-APOM: (a) image du panache à $550 \mathrm{~nm}$, (b) carte de la fraction en suie (\%), (c) carte des rayons effectifs $(\mu \mathrm{m}),(\mathrm{d})$ carte de l'épaisseur optique à $550 \mathrm{~nm}$, (e) carte de l'albédo de diffusion simple à $550 \mathrm{~nm},(\mathbf{f})$ carte du coefficient d'asymétrie (lié à la fonction de phase) à $550 \mathrm{~nm}$.

\section{Télédétection des aérosols}

De manière générale, l'estimation des aérosols à partir d'images est un problème délicat car la contribution du signal aérosol dans le signal total est faible et doit être séparée de celle du sol. Ceci est particulièrement difficile sur les terres émergées à cause de l'hétérogénéité spatiale des surfaces terrestres.

Pour résoudre ce problème, une approche souvent retenue est l'approche dite des surfaces sombres développée par Kaufman et al. [3]. Elle consiste à observer des pixels pour lesquels le sol a une réflectance faible dans le visible (eau, végétation dense). Pour ces pixels, le signal de luminance enregistré provient essentiellement de la luminance atmosphérique: la contribution des aérosols est donc maximale. Ensuite, à $0,49 \mu \mathrm{m}$ et $0,66 \mu \mathrm{m}$, l'épaisseur optique des aérosols est déduite du signal enregistré à l'aide d'une inversion par look-up table / LUT (comparaison du signal enregistré à des signaux simulés en faisant varier les paramètres

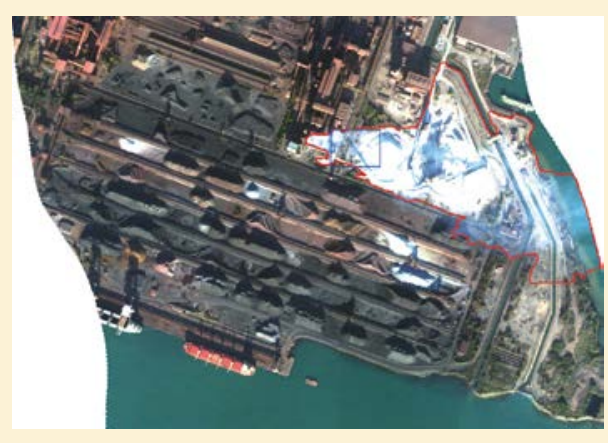

aérosols). Enfin, la loi d'Angström permet d'extrapoler l'épaisseur optique sur l'ensemble du spectre visible. Cette méthode permet ainsi de déterminer le type des aérosols (choisi parmi un nombre restreint de modèles dits standards); tous les pixels de l'image seront ensuite supposés contenir ce même type d'aérosols. Une autre limitation réside dans la nécessité d'avoir des zones sombres sur l'image étudiée.

\section{Les aérosols de panache}

Cependant, les aérosols de panaches ne peuvent pas être correctement décrits par des modèles standards. Il convient alors de développer des modèles adaptés, qui permettent de prendre en compte la variabilité de leur composition dans l'image. Le cas des aérosols anthropiques est encore plus délicat car leurs indices de réfraction sont beaucoup moins bien connus que ceux des aérosols naturels ce qui ne permet pas d'aboutir à un modèle physique permettant de décrire, de manière

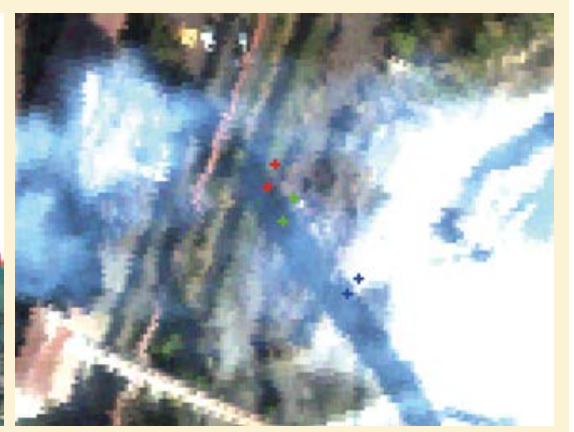

Figure 3. (a) Image hyperspectrale CASI $\left(\lambda_{B}=458 \mathrm{~nm}, \lambda_{V}=549 \mathrm{~nm}, \lambda_{R}=640 \mathrm{~nm}\right)$ acquise le 28 octobre 2010 sur le site d'une industrie métallurgique à Fos-sur-Mer (la ligne rouge indique le contour du panache). (b) Zoom correspondant au rectangle bleu et localisation des couples de pixels sélectionnés (marqués en rouge, vert et bleu). générale, les particules émises par les industries. En effet, celles-ci sont de nature très variable, d'une part parce que leur composition dépend très fortement du type d'industrie dont elles proviennent, d'autre part parce que leurs propriétés varient sensiblement avec la distance du point d'émission, $\mathrm{du}$ fait des nombreuses interactions avec les gaz atmosphériques.

Aussi, des méthodes spécifiques à la caractérisation des aérosols de panaches ont récemment été développées.

\section{Panaches optiquement denses}

La méthode L-APOM (LUT - aerosol plume optical model) décrite par Alakian et al. [4] permet une caractérisation fine des propriétés optiques et microphysiques de panaches optiquement denses dont le type est connu a priori.

Elle permet en particulier de déterminer la concentration des particules dans un panache de feu de végétation, mais également leur taille et la proportion de suie dans le mélange d'aérosols (figure 2). Elle utilise une base de spectres de luminance générée par un code de transfert radiatif pour une atmosphère contenant une couche d'aérosols ayant différentes propriétés microphysiques. Ensuite, pixel à pixel, le spectre enregistré par le capteur est comparé à chacun des spectres contenus dans la base préalablement générée. On retient alors l'élément de la base qui, lorsqu'on le compare au spectre mesuré, minimise l'erreur quadratique. On remonte ensuite aux propriétés microphysiques des aérosols en retrouvant les paramètres d'entrée qui ont permis de générer ce spectre. Des contraintes spatiales ont également été implémentées pour limiter les disparités dans les propriétés obtenues pour des pixels proches.

\section{Panaches industriels à faibles épaisseurs optiques}

Un panache émis par une usine est généralement d'une épaisseur optique bien plus faible que les panaches volcaniques ou de feu de végétation (figure 3). La séparation, dans le signal de luminance, entre la contribution du sol et des aérosols est donc difficile. 


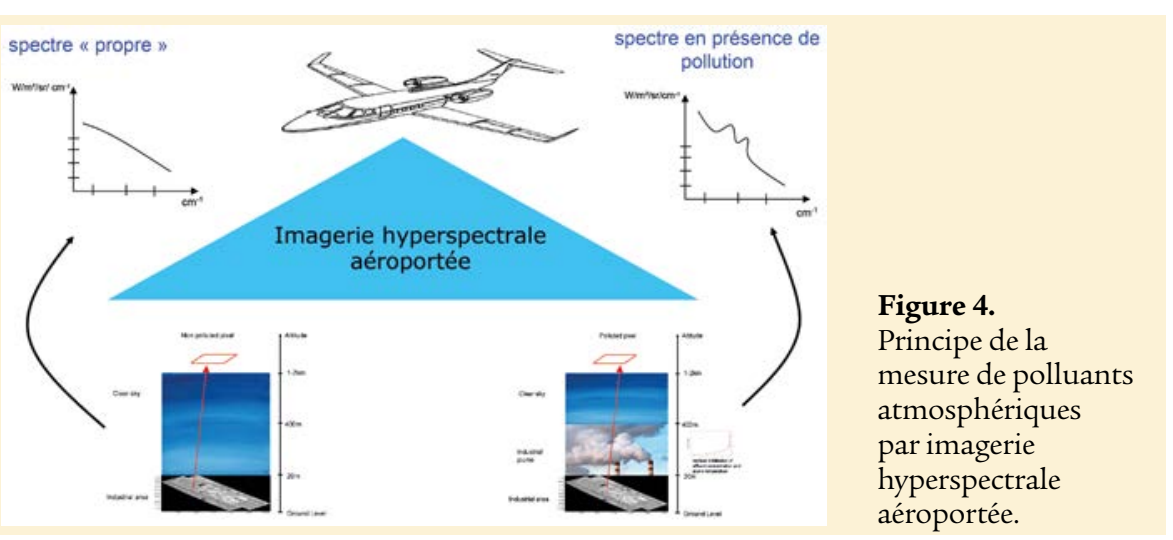

La méthode EARTH [5] est particulièrement adaptée aux panaches peu denses optiquement. Elle consiste en l'estimation de deux propriétés optiques des aérosols, l'épaisseur optique et le produit de l'albédo de diffusion simple et de la fonction de phase, à partir de couples de pixels situés dans le panache, sur des transitions entre deux types de sol ou entre une surface à l'ombre et la même surface au soleil. À partir de ces propriétés estimées, le type d'aérosol est déterminé par comparaison avec des propriétés préalablement calculées pour différents types d'aérosols et stockées dans une LUT.

Cette méthode de détermination du type d'aérosols présents dans un panache, est particulièrement adaptée aux effluents industriels, et ouvre donc de nouvelles perspectives, notamment pour le contrôle de pollution par télédétection. De plus, cette méthode peut être couplée avec une autre méthode d'inversion (par exemple L-APOM) pour obtenir, une fois le modèle d'aérosol connu, des cartes de propriétés optiques et microphysiques.

\section{Caractérisation de polluants gazeux}

\section{Principe de la mesure}

La figure 4 illustre le concept de caractérisation de polluants atmosphériques par mesure différentielle. Cette mesure différentielle permet de remonter aux propriétés spectrales du panache gazeux. En effet, l'impact radiatif d'un gaz provient de ses différents modes de vibration-rotation et des collisions intermoléculaires entrainant des échanges d'énergie avec son environnement dans le domaine optique et principalement dans le domaine infrarouge.

Les imageurs optiques hyperspectraux ont des résolutions spectrales comprises entre 5 et $15 \mathrm{~cm}^{-1}$ suivant les instruments dans la gamme 3-12 $\mu \mathrm{m}$ pour un bruit instrumental compris entre 0,3 et $0,05 \mathrm{~K}$ en température de brillance. Comme la gamme de résolution spectrale ne permet pas de résoudre les raies moléculaires, les gaz d'intérêt sont modélisés uniquement par leur section efficace spectrale. Ces données sont obtenues par mesure de transmission par interférométrie infrarouge en laboratoire; leur unité est le $\mathrm{m}^{-1} \mathrm{ppmv}^{-1}$. Il s'agit d'une épaisseur optique normalisée en épaisseur de gaz et en concentration volumique.

La figure 5 montre ainsi un exemple de signal différentiel exprimé en température de brillance pour un panache fin de dioxyde de soufre et pour un panache d'éthylène. Ces données sont convoluées aux résolutions spectrales

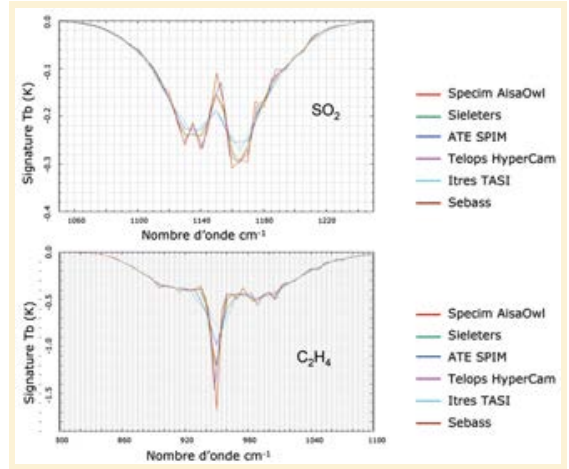

Figure 5. Exemples de signatures différentielles en température de brillance $(\mathrm{Tb})$ caractéristiques du $\mathrm{SO}_{2}$ (haut) et de l'éthylène (bas) vues par les principaux capteurs hyperspectraux infrarouge actuels.

\section{Analyseur de spectre osa20}

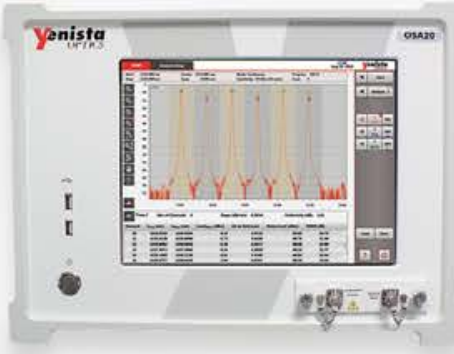

Rapide et précis

Plage spectrale $1250-1700 \mathrm{~nm}$

Vitesse maximale $2000 \mathrm{~nm} / \mathrm{s}$

\section{Résolution spectrale 20 pm}

Précision $\lambda$

$\pm 10 \mathrm{pm} / 1500-1640 \mathrm{~nm}$ $\pm 25 \mathrm{pm} / 1250-1700 \mathrm{~nm}$

\section{Ergonomique}

- Ecran tactile multipoints

- Modes d'utilisation par application

- Librairie de fonctions $d^{\prime}$ analyse

Découvrez toutes les autres fonctionnalités sur www.yenista.com/osa20

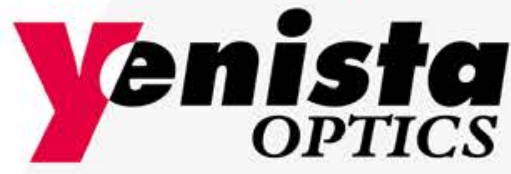

Tél. : +33 (0)296483716 sales-emea@yenista.com www.yenista.com 


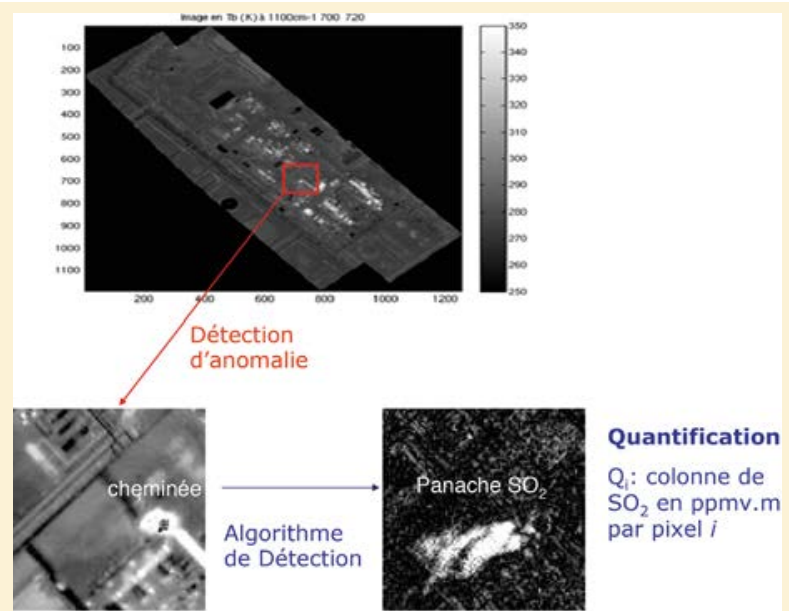

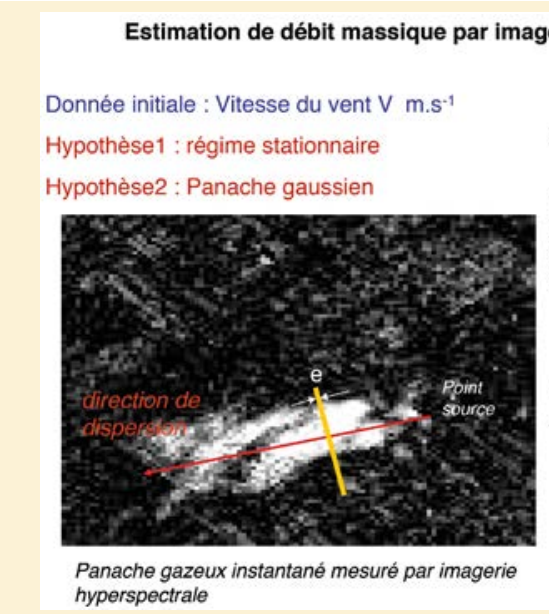
hyperspectrale

Figure 6. Étapes du processus de caractérisation de gaz. Image en haut: image infrarouge à $9 \mu \mathrm{m}$ d'un site industriel. Image en bas à gauche: vignette de la même image hyperspectrale où le maximum d'intensité en température de brillance (zone blanche au centre) reflète la présence d'une source de chaleur intense: sortie de cheminée industrielle. Image en bas à droite: même vignette pour laquelle un filtre GLRT adapté au $\mathrm{SO}_{2}$ a été appliqué, le panache de $\mathrm{SO}_{2}$ est ainsi mis en évidence, on peut alors estimer ses propriétés.

Mesures : $Q_{i}$ en ppmv.m par pixel $i$

Intégration sur panache contenu sur une section S orthogonale à la direction de propagation, d'épaisseur e (pixels jaunes)

$$
Q_{s}=\sum Q_{i}
$$

Calcul du débit massique pour la section S:

$$
D=\frac{M_{\text {suz }}}{22.46} \cdot \frac{Q_{s}}{e} \cdot V
$$

Figure 7. Estimation de débit massique par imagerie hyperspectrale. des principaux imageurs hyperspectraux existant à ce jour dans le domaine 8-12 $\mu \mathrm{m}$. Il apparaît ici, tout d'abord, que le seuil de détection d'un constituant gazeux est propre à chaque gaz et est d'autant plus faible que la résolution spectrale de l'imageur utilisé est haute.

\section{De la détection de gaz au calcul de flux massique}

Les méthodes de détection ont pour objectif de faire ressortir les pixels ayant une caractéristique spectrale et/ ou spatiale particulière dans la scène. L'idée ici est d'associer à chaque pixel un score qui reflète à la fois sa dissimilarité avec le comportement moyen des pixels et une similarité avec une signature de gaz connu. Le comportement moyen des pixels est couramment caractérisé par le spectre moyen et la matrice de variance covariance contenant, pour chaque bande, l'information liée à l'écart type et les corrélations spectrales entre les bandes.

Les deux méthodes les plus couramment utilisées sont le filtrage adapté et la méthode du maximum de vraisemblance généralisé dont le principe est d'utiliser des spectres pré-calculés dans diverses conditions environnementales, et de rechercher dans les images les pixels dont le comportement spectral se rapproche le plus des espaces spectraux ainsi créés (figure 6).
L'équation du transfert radiatif est non linéaire et le problème inverse est indéterminé: plusieurs couples de solutions conduisent au même résultat. Cependant autour d'un point de fonctionnement, il est possible sous certaines hypothèses de proposer un modèle linéaire du type $\mathrm{Y}=\mathrm{JX}$ où $\mathrm{Y}$ est la mesure, $\mathrm{J}$ est le modèle et $\mathrm{X}$ la grandeur accessible et pour lequel il existe une solution exacte et unique. Dans le cas de la caractérisation de gaz, le point de fonctionnement nominal de référence correspond à un pixel présentant un fond similaire au pixel pollué par un panache gazeux. C'est la différence de luminance entre ces deux pixels qu'il est possible alors d'approcher de façon linéaire, un formalisme détaillé et complet est proposé par Young [6].

La grandeur accessible correspond alors à la quantité de gaz intégré en ppmv m (figure 6) le long de la ligne de visée, pondérée par la différence de température entre le sol et le panache gazeux. Si le panache est plus froid que le sol, on observe un signal d'absorption (cas présenté à la figure 5). Dans le cas contraire, on observe l'émission du panache.

Lafigure 7 présente enfin comment, à partir de la mesure d'une colonne intégrée de gaz pixel par pixel, d'hypothèses sur la dispersion du panache, et de la connaissance de la donnée de vent, il est alors possible de remonter au débit massique des divers polluants gazeux.

\section{RÉFÉRENCES}

[1] Équipe de rédaction principale, R. Pachauri, A. Reisinger, Bilan 2007 des changements climatiques: Rapport de synthèse, Groupe d'experts intergouvernemental sur l'évolution du climat, Organisation météorologique mondiale (OMM), Tech. Rep., 2008.

[2] L. Rousset-Rouviere, C. Coudrain, S. Fabre, N. Guérineau, J.P. Bruyant, I. Baarstad, T. Løke, A. Fridman, and S. Blaaberg, SYSIPHE: The new-generation airborne remote sensing system, SPIE Remote Sensing, 26-27 September 2012, Edinburgh United Kingdom, Proceedings of SPIE, vol. 8532.

[3] Y.J. Kaufman, A. Wald, L. Remer, B. Gao, R. Li, and L. Flynn, The MODIS 2.1- $\mu m$ channelcorrelation with visible reflectance for use in remote sensing of aerosol, IEEE Trans. Geosci. Remote Sens., vol. 35, pp. 1286-1297, 1997.

[4] A. Alakian, R. Marion, and X. Briottet, Retrieval of microphysical and optical properties in aerosol plumes with hyperspectral imagery: L-APOM method, Remote Sens. Environ., vol. 113, pp. 781-793, 2009.

[5] A. Deschamps, Caractérisation de panaches industriels par imagerie hyperspectrale, Thèse de Doctorat, Université Pierre et Marie Curie, 19 décembre 2012.

[6] Young, Detection and quantification of gases in industrial-stack plumes using thermal-infrared byperspectral imaging, 10 February 2002, Aerospace Corporation. 